\title{
Pediatric Extubation Readiness: Faith-Based Practice or Amenable to Standardization?
}

Faith, defined as a firm belief in something for which there is no proof, is in contrast to observation and inference, in which we learn and make a conclusion about what the evidence tells us. If we fail to continue to observe and change our conclusions, then one can fall into a practice of faith. Is there opportunity to obtain new evidence, and move away from attempting to apply adult concepts to the weaning of mechanical ventilation in pediatrics, and form a basis for good practice? One way to approach this is to assess practice, identify what we do, apply respiratory developmental physiology, and contrast that with what we know.

In this issue of Respiratory CARE, Mhanna et al provide an overview of what pediatric critical care providers think should be done prior to extubation in pediatric patients, as well as probe what they actually do. ${ }^{1}$ As with all surveys, we must take the answers with some skepticism, as the responses may not reflect true practice, but it does give us a perspective on current thinking among pediatric intensivists. Mhanna et al found that checking for an air leak, assessing secretion suctioning frequency, and sedation are

See the Original Study on Page 334

the top items intensivists consider, and most do not consider airway occlusion pressure or the rapid shallow breathing index. Spontaneous breathing trials (SBTs) are performed mainly with pressure support. The majority of intensivists are looking for an air leak at $20-30 \mathrm{~cm} \mathrm{H}_{2} \mathrm{O}$ peak airway pressure and a suctioning frequency of around every $2-4$ hours. An interesting pearl in the survey is that there was no correlation based on institution, which suggests that individual clinician interpretation and approach somewhat overrides institutional practice.

No pediatric study has yet to improve upon the standard of when a clinician "feels" the patient is ready for extubation. The only prospective multi-center weaning and extubation trial in pediatrics found no difference in success based on approach, ${ }^{2}$ despite a much higher extubation failure rate than previously described in another large multicenter cohort (17\% vs 6\%). ${ }^{3}$ Studies of extubation readiness tests start from a baseline in which the clinician already feels the patient is ready to extubate, and it is difficult to determine what value the various assessments or tests add to the decision to extubate. ${ }^{4}$

If no approach is better then the clinician's clinical assessment, how does the clinician determine the patient is ready? Mhanna highlights the standard approach: to assess leak, secretions, do an SBT, and assess sedation. How do these stand up to the evidence?

The leak test reliability is positional, requiring neutral position and muscle relaxation, ${ }^{5}$ and yet in the pediatric ICU this may not be universally determined in this manner. There has been no consistency in the results of studies of cuff leak tests for predicting post-extubation failure or stridor, nor what level of leak or at what pressure is best to ensure success. ${ }^{6,7}$

The issue of tracheal secretions has never been studied in children. Do children do better with frequent suctioning if they are extubated or intubated? Is it better to cough it out or suction it out? Surely context matters: frequent suctioning on minimal oxygenation and ventilation means something different than higher ventilation needs. Is it secretion suctioning frequency, volume obtained, thickness, or color that is the critical piece in extubation failure? This remains a subject open for evaluation, and while observations of secretions have made it into clinical practice or just been adopted from adult practice or our mentors, no one has yet attempted to study this in a scientific fashion in pediatrics.

SBTs are feasible in children and may decrease the duration of mechanical ventilation in children, compared to clinician "feeling" of readiness. ${ }^{8}$ The preference for SBT with pressure support has become mainstream, despite no clear evidence of benefit over CPAP or T-piece alone, perhaps over continued misconceptions about endotracheal tube resistance. ${ }^{2}$ In addition, there is less reliance by intensivists on sedation scores when considering extubation, perhaps because it is an assessment at an individual point in time versus looking clinically at the patient to see if at that point the patient is awake.

One item that is poorly investigated in pediatrics in weaning trials is if there are developmental physiology factors based on a child's age and size that are relevant extubation predictors. Is comparing a 3-month-old and a 15 -year-old for weaning using the same strategy valid? Factors like normal oxygen saturation range, carbon diox- 
ide response curve, developmental changes in the trachea, chest wall, and diaphragmatic apposition in the chest may all play a role in extubation success. One might find a leak test is valid for a 15-year-old and unnecessary in a 3-monthold old, or vice versa. This may be the key to determining what can be extrapolated from adults, and what needs to be individually tailored based on growth-dependent respiratory physiology.

Overall, the data remain sparse about how to best approach extubation in pediatric critical care. "Faith" in one approach has yet to be supplanted by evidence in pediatric weaning of mechanical ventilation.

\section{Brian M Cummings MD}

Natan Noviski MD

Division of Pediatric Critical Care Medicine Massachusetts General Hospital

Boston, Massachusetts

The authors have disclosed no conflicts of interest.

Correspondence: Natan Noviski MD, Pediatric Critical Care Medicine, Massachusetts General Hospital, Harvard Medical School, 175 Cambridge Street, CPZS-524, Boston MA 02114. E-mail: nnoviski@partners.org.

DOI: $10.4187 /$ respcare.03103

\section{REFERENCES}

1. Mhanna MJ, Anderson IM, Iyer NP, Baumann A. The use of extubation readiness parameters: a survey of pediatric critical care physicians. Respir Care 2014;59(3):334-339.

2. Randolph AG, Wypij D, Venkataraman ST, Hanson JH, Gedeit RG, Meert KL, et al. Effect of mechanical ventilator weaning protocols on respiratory outcomes in infants and children: a randomized controlled trial. JAMA 2002;288(20):2561-2568.

3. Kurachek SC, Newth CJ, Quasney MW, Rice T, Sachdeva RC, Patel $\mathrm{NR}$, et al. Extubation failure in pediatric intensive care: a multiplecenter study of risk factors and outcomes. Crit Care Med 2003; 31(11):2657-2664.

4. Chavez A, de la Cruz R, Zaritsky A. Spontaneous breathing trial predicts successful extubation in infants and children. Pediatr Crit Care Med 2006;7(4):324-328.

5. Finholt DA, Henry DB, Raphaely RC. Factors affecting leak around tracheal tubes in children. Can Anaesth Soc J 1985;32(4):326-329.

6. Mhanna MJ, Zamel YB, Tichy CM, Super DM. The "air leak" test around the endotracheal tube, as a predictor of postextubation stridor, is age dependent in children. Crit Care Med 2002;30(12):2639-2643.

7. Wratney AT, Benjamin DK Jr, Slonim AD, Hamel DS, Cheifetz IM. The endotracheal tube air leak test does not predict extubation outcome in critically ill pediatric patients. Pediatr Crit Care Med 2008; 9(5):490-496.

8. Foronda FK, Troster EJ, Farias JA, Barbas CS, Ferraro AA, Faria LS, et al. The impact of daily evaluation and spontaneous breathing test on the duration of pediatric mechanical ventilation: a randomized controlled trial. Crit Care Med 2011;39(11):2526-2533. 